\title{
Concepts of Mesoscopic Continuum Physics With Application to Biaxial Liquid Crystals ${ }^{1)}$
}

\author{
W. Muschik, H. Ehrentraut, C. Papenfuss \\ Institut für Theoretische Physik, Technische Universität Berlin, Berlin, Germany \\ Registration Number 865
}

\author{
Aus einem kleinen Gebüsch \\ springt oftmals ein großer Hase
}

\begin{abstract}
The mesoscopic concept in continuum mechanics consists of extending the domain of the balance equations by the set of mesoscopic variables and of introducing a local distribution function of these variables as a statistical element. The balance equations defined on this extended domain and an example concerning liquid crystals of biaxial molecules are discussed.
\end{abstract}

\section{Introduction}

Continuum mechanics is based on the balance equations of mass, momentum, angular momentum or spin, total or kinetic energy, and internal energy. Additionally one has to consider the balance of entropy for taking into account the second law. In non-relativistic physics all these balances are defined on time and position $(\boldsymbol{x}, t)$. Beyond the quantities whose balance equations are mentioned above, complex materials need more variables for their unique description. Examples for these additional quantities are internal variables [1] [2], order and damage parameters [3] [4], Cosserat triads [5], directors [6] [7], and alignment and conformation tensors [8] [9]. In principle there are two possibilities to include these additional quantities into the continuum theoretical description: One can introduce additional fields and their balance equations defined on $(\boldsymbol{x}, t) \in \mathbb{R}^{3} \times \mathbb{R}^{1}$, or the additional quantities are introduced as variables extending $\mathbb{R}^{3} \times \mathbb{R}^{1}$ to the so-called mesoscopic space on which now the balances of mass, momentum, etc., are defined. The first possibility for describing complex materials by introducing additional fields has a long history in

${ }^{1)}$ Dedicated to Prof. P. Haupt on occasion of his $60^{\text {th }}$ birthday.

Partly delivered at the International Seminar Geometry, Mechanics, and Microstructure, Laboratoire de Modélisation en Mécanique, Université Pierre et Marie Curie, Paris VI, 1997. 
continuum mechanics. Starting out with the first contribution of the Cosserat brothers [10] [11] the development of mechanics of generalized continua is lasting since today [12] [13] [14]. The second possibility introducing the mesoscopic space is called the mesoscopic concept which stems historically from the theory of liquid crystals [3] [6] [7] [15] [16] [17] [8] by taking the orientation distribution function of the molecules into consideration [18] [19] [20]. This mesoscopic description of complex materials is discussed in more detail in the next section.

\section{Mesoscopic Concept}

As discussed above the mesoscopic concept introduces the mesoscopic space

$$
(\boldsymbol{m}, \boldsymbol{x}, t) \in \mathscr{M} \times \mathbb{R}^{3} \times \mathbb{R}^{1}
$$

on which the balances are defined. Here $\boldsymbol{m} \in \mathscr{M}$ is a set of mesoscopic variables which is an element of a suitable manifold $\mathscr{M}$ on which an integration can be defined.

An example for $\boldsymbol{m}$ is the microscopic director $\boldsymbol{n}$ in mesoscopic liquid crystal theory [19]. This microscopic director is defined as a unit vector pointing into the temporary direction of a needle-shaped rigid particle, or, if the particle is of a plane shape, the microscopic director is perpendicular to the particle. Because the microscopic director is defined on a molecular level it is not a macroscopic field $\boldsymbol{d}(\boldsymbol{x}, t)$ describing the mean orientation, but a mesoscopic variable. Here "mesoscopic" means that the level of description is finer than the macroscopic one, but that no microscopic concepts such as molecular interactions or potentials are used. In this example the manifold $\mathscr{M}$ in (1) is the 2-dimensional unit sphere $\mathbb{S}^{2}$.

Beyond the use of additional variables $\boldsymbol{m}$ the mesoscopic concept introduces a statistical element, the so-called mesoscopic distribution function (MDF) $f(\boldsymbol{m}, \boldsymbol{x}, t)$ generated by the different values of the mesoscopic variables of the molecules in a volume element

$$
f(\boldsymbol{m}, \boldsymbol{x}, t) \equiv f(\cdot), \quad(\cdot) \equiv(\boldsymbol{m}, \boldsymbol{x}, t) \in \mathscr{M} \times \mathbb{R}^{3} \times \mathbb{R}^{1}
$$

The MDF is defined on the mesoscopic space $\mathscr{M} \times \mathbb{R}^{3} \times \mathbb{R}^{1}$ describing the distribution of $\boldsymbol{m}$ in a volume element around $\boldsymbol{x}$ at time $t$, and therefore it is always normalized

$$
\int f(\boldsymbol{m}, \boldsymbol{x}, t) d \mathscr{M}=1 .
$$

Now the fields as mass density, momentum density, specific internal energy, etc. are defined on the mesoscopic space. For distinguishing these fields from the usual, macroscopic ones we add the word "mesoscopic". Consequently the mesoscopic mass density is defined by

$$
\varrho(\cdot):=\varrho(\boldsymbol{x}, t) f(\cdot) .
$$


Here $\varrho(\boldsymbol{x}, t)$ is the macroscopic mass density. By use of (3) we obtain

$$
\varrho(\boldsymbol{x}, t)=\int \varrho(\boldsymbol{m}, \boldsymbol{x}, t) d \mathscr{M} .
$$

This equation shows, that the system can be formally treated as a mixture by regarding all particles in a volume element of the same mesoscopic variables as one component of the system having the partial density $\varrho(\cdot)$ [20]. Thus the MDF results as the fraction of the mass density belonging to one component characterized by the same mesoscopic variables over the total mass density of the mixture. Here the "component index" $\boldsymbol{m}$ is a continuous one. Because mixture theory is well developed [21], [22] mesoscopic balance equations can be written down very easily [23], what we will do in the next section.

Other mesoscopic fields defined on the mesoscopic space are the mesoscopic material velocity $\boldsymbol{v}(\cdot)$ of the particles belonging to the mesoscopic variable $\boldsymbol{m}$ at time $t$ in a volume element around $\boldsymbol{x}$, the external mesoscopic acceleration $\boldsymbol{k}(\cdot)$, the mesoscopic stress tensor $\boldsymbol{T}(\cdot)$, and the mesoscopic heat flux density $\boldsymbol{q}(\cdot)$, etc. The balance equations belonging to these mesoscopic fields are discussed in the next section.

\section{Mesoscopic Balances}

\subsection{Global mesoscopic balances}

First of all we write down a general global mesoscopic balance equation [19] which has the following form

$$
\begin{aligned}
& \frac{d}{d t} \int \varrho(\cdot)[\phi(\cdot) \boldsymbol{v}(\cdot)+\boldsymbol{\Phi}(\cdot)] d \mathscr{M} d^{3} x=\boldsymbol{Z}^{t o t}, \\
& \boldsymbol{Z}^{\text {tot }}=\int\left[\boldsymbol{\Sigma}(\cdot)+\nabla_{x} \cdot \boldsymbol{S}(\cdot)+\nabla_{m} \cdot \boldsymbol{R}(\cdot)\right] d \mathscr{M} d^{3} x .
\end{aligned}
$$

Here $\phi(\cdot)$ is the convective part of the balance, $\boldsymbol{\Phi}(\cdot)$ its conductive part, $\boldsymbol{\Sigma}(\cdot)$ the volume part, and $\boldsymbol{S}(\cdot)$ and $\boldsymbol{R}(\cdot)$ are the fluxes through the surface in position space and on the manifold $\mathscr{M}$, respectively. The total change $\boldsymbol{Z}^{\text {tot }}$ is the sum of the production and the total flux over the boundary and it belongs to the spatial and to the mesoscopic part of the mesoscopic space. The interpretation of the balanced quantity

$$
\boldsymbol{X}(\cdot):=\varrho(\cdot)[\phi(\cdot) \boldsymbol{v}(\cdot)+\boldsymbol{\Phi}(\cdot)]
$$

in (6) depends on $\phi(\cdot)$ and on $\boldsymbol{\Phi}(\cdot)$ which are functions on the mesoscopic space. Therefore the meaning of the balanced quantity is also determined by the meaning of the set of mesoscopic variables $\boldsymbol{m}$. Consequently an interpretation of a global mesoscopic balance equation can be given, if the physical character of the set of mesoscopic variables is fixed. In Section 6 we will treat an example chosen from the field of liquid crystals. 
The global mesoscopic balance equation (6) can be transformed into a mesoscopic one which is local in position, time, and in the set of mesoscopic variables. For this purpose we need a generalized Reynolds' transport theorem [19].

$$
\begin{aligned}
& \frac{d}{d t} \int \boldsymbol{\Phi}(\cdot) d \mathscr{M} d^{3} x \\
& \quad=\int\left[\frac{\partial}{\partial t} \boldsymbol{\Phi}(\cdot)+\nabla_{x} \cdot[\boldsymbol{v}(\cdot) \boldsymbol{\Phi}(\cdot)]+\nabla_{m} \cdot[\boldsymbol{u}(\cdot) \boldsymbol{\Phi}(\cdot)]\right] d \mathscr{M} d^{3} x .
\end{aligned}
$$

Here the independent field $\boldsymbol{u}(\cdot)$, defined on the mesoscopic space, describes the change in time of the set of mesoscopic variables: With respect to $\boldsymbol{m}$ the mesoscopic change velocity $\boldsymbol{u}(\cdot)$ is the analogue to the mesoscopic material velocity $\boldsymbol{v}(\cdot)$ referring to $\boldsymbol{x}$ : If a molecule is characterized by $(\boldsymbol{m}, \boldsymbol{x}, t)$, then for $\Delta t \rightarrow 0$ it is characterized by $(\boldsymbol{m}+\boldsymbol{u}(\cdot) \Delta t, \boldsymbol{x}+\boldsymbol{v}(\cdot) \Delta t, t+\Delta t)$. Besides the usual gradient also the gradient with respect to the set of mesoscopic variables appear. By use of (9) we can transform the global mesoscopic balance equation (6) as usual into a local one.

\subsection{Local mesoscopic balances}

Presupposing the global balances we obtain by use of (9) local balance equations [24] which are defined on the mesoscopic space and which are therefore denoted as local mesoscopic balances. The general structure of these balances for the balanced quantity (8) is

$$
\frac{\partial}{\partial t} \boldsymbol{X}(\cdot)+\nabla_{x} \cdot[\boldsymbol{v}(\cdot) \boldsymbol{X}(\cdot)-\boldsymbol{S}(\cdot)]+\nabla_{m} \cdot[\boldsymbol{u}(\cdot) \boldsymbol{X}(\cdot)-\boldsymbol{R}(\cdot)]=\boldsymbol{\Sigma}(\cdot) .
$$

As already discussed the special balances are obtained by a special identification of $\phi(\cdot)$ and $\boldsymbol{\Phi}(\cdot)$ by which an interpretation of $\boldsymbol{S}(\cdot)$ and $\boldsymbol{R}(\cdot)$ follows. In the special case of the balance of mass these four quantities are independent of any interpretation of $\boldsymbol{m}$, because whatever $\boldsymbol{m}$ may be, the mass balance must be valid. We obtain the mesoscopic balances by the subsequent identifications [23]:

Mass

$$
\begin{aligned}
& \phi(\cdot) \equiv 0, \quad \boldsymbol{\Phi}(\cdot) \equiv 1, \quad \boldsymbol{\Sigma}(\cdot) \equiv 0, \\
& \boldsymbol{S}(\cdot) \equiv \mathbf{0}, \quad \boldsymbol{R}(\cdot) \equiv \mathbf{0} . \\
& \frac{\partial}{\partial t} \varrho(\cdot)+\nabla_{x} \cdot\{\varrho(\cdot) \boldsymbol{v}(\cdot)\}+\nabla_{m} \cdot\{\varrho(\cdot) \boldsymbol{u}(\cdot)\}=0 .
\end{aligned}
$$

Momentum

$$
\begin{aligned}
\phi(\cdot) \equiv 1, \quad \boldsymbol{\Phi}(\cdot) \equiv \mathbf{0}, \quad \boldsymbol{\Sigma}(\cdot) \equiv \varrho(\cdot) \boldsymbol{k}(\cdot) \\
\boldsymbol{S}(\cdot) \equiv \boldsymbol{T}^{\top}(\cdot), \quad \boldsymbol{R}(\cdot) \equiv \mathscr{T}^{\top}(\cdot), \\
\frac{\partial}{\partial t}[\varrho(\cdot) \boldsymbol{v}(\cdot)]+\nabla_{x} \cdot\left[\boldsymbol{v}(\cdot) \varrho(\cdot) \boldsymbol{v}(\cdot)-\boldsymbol{T}^{\top}(\cdot)\right] \\
+\nabla_{m} \cdot\left[\boldsymbol{u}(\cdot) \varrho(\cdot) \boldsymbol{v}(\cdot)-\mathscr{T}^{\top}(\cdot)\right]=\varrho(\cdot) \boldsymbol{k}(\cdot) .
\end{aligned}
$$


Here $\boldsymbol{k}(\cdot)$ is the external acceleration, $\boldsymbol{T}^{\top}(\cdot)$ the transposed Cauchy stress tensor, and $\mathscr{T}^{\top}(\cdot)$ the transposed stress tensor on $\mathscr{M}$.

Angular Momentum

$$
\begin{aligned}
& \phi(\cdot) \equiv \boldsymbol{x} \times, \quad \boldsymbol{\Phi}(\cdot) \equiv \boldsymbol{s}(\cdot), \\
& \boldsymbol{\Sigma}(\cdot) \equiv \boldsymbol{x} \times \varrho(\cdot) \boldsymbol{k}(\cdot)+\varrho(\cdot) \boldsymbol{g}(\cdot), \\
& \boldsymbol{S}(\cdot) \equiv[\boldsymbol{x} \times \boldsymbol{T}(\cdot)]^{\top}+\boldsymbol{W}^{\top}(\cdot), \\
& \boldsymbol{R}(\cdot) \equiv[\boldsymbol{x} \times \mathscr{T}(\cdot)]^{\top}+\mathscr{W}^{\top}(\cdot), \\
& \boldsymbol{M}(\cdot):=\boldsymbol{x} \times \boldsymbol{v}(\cdot)+\boldsymbol{s}(\cdot), \\
& \frac{\partial}{\partial t}[\varrho(\cdot) \boldsymbol{M}(\cdot)]+\nabla_{x} \cdot\left[\boldsymbol{v}(\cdot) \varrho(\cdot) \boldsymbol{M}(\cdot)-(\boldsymbol{x} \times \boldsymbol{T}(\cdot))^{\top}-\boldsymbol{W}^{\top}(\cdot)\right] \\
& \quad+\nabla_{m} \cdot\left[\boldsymbol{u}(\cdot) \varrho(\cdot) \boldsymbol{M}(\cdot)-(\boldsymbol{x} \times \mathscr{T}(\cdot))^{\top}-\mathscr{W}^{\top}(\cdot)\right] \\
& \quad=\varrho(\cdot) \boldsymbol{x} \times \boldsymbol{k}(\cdot)+\varrho(\cdot) \boldsymbol{g}(\cdot) .
\end{aligned}
$$

Here $s(\cdot)$ is the vector of mesoscopic specific spin, $g(\cdot)$ the vector of mesoscopic angular momentum exerted by the external forces on $\boldsymbol{m}$, the tensor $\boldsymbol{W}(\cdot)$ is the mesoscopic surface torque, and $\mathscr{W}(\cdot)$ is the analogue to $\mathscr{T}(\cdot)$ acting on the mesoscopic variable $\boldsymbol{m}$.

\section{Total Energy}

$$
\begin{aligned}
\phi(\cdot) & \equiv(1 / 2) \boldsymbol{v}(\cdot), \quad \boldsymbol{\Phi}(\cdot) \equiv(1 / 2) \boldsymbol{s}(\cdot) \cdot \Theta^{-1} \cdot \boldsymbol{s}(\cdot)+\varepsilon(\cdot), \\
\boldsymbol{\Sigma}(\cdot) & \equiv \varrho(\cdot) \boldsymbol{k}(\cdot) \cdot \boldsymbol{v}(\cdot)+\varrho(\cdot) \boldsymbol{g}(\cdot) \cdot \Theta^{-1} \cdot \boldsymbol{s}(\cdot)+\varrho(\cdot) r(\cdot), \\
\boldsymbol{S}(\cdot) & \equiv \boldsymbol{T}(\cdot)^{\top} \cdot \boldsymbol{v}(\cdot)+\boldsymbol{W}(\cdot)^{\top} \cdot \Theta^{-1} \cdot \boldsymbol{s}(\cdot)+\boldsymbol{q}(\cdot), \\
\boldsymbol{R}(\cdot) & \equiv \mathscr{T}(\cdot)^{\top} \cdot \boldsymbol{v}(\cdot)+\mathscr{W}(\cdot)^{\top} \cdot \Theta^{-1} \cdot \boldsymbol{s}(\cdot)+\boldsymbol{Q}(\cdot), \\
e(\cdot) & :=\frac{1}{2} \boldsymbol{v}^{2}(\cdot)+\frac{1}{2} \boldsymbol{s}(\cdot) \cdot \Theta^{-1} \cdot \boldsymbol{s}(\cdot)+\varepsilon(\cdot), \\
\frac{\partial}{\partial t}[\varrho(\cdot) e(\cdot)] & \\
& +\nabla_{x} \cdot\left[\boldsymbol{v}(\cdot) \varrho(\cdot) e(\cdot)-\boldsymbol{T}(\cdot)^{\top} \cdot \boldsymbol{v}(\cdot)-\boldsymbol{W}(\cdot)^{\top} \cdot \Theta^{-1} \cdot \boldsymbol{s}(\cdot)+\boldsymbol{q}(\cdot)\right] \\
& +\nabla_{m} \cdot\left[\boldsymbol{u}(\cdot) \varrho(\cdot) e(\cdot)-\mathscr{T}(\cdot)^{\top} \cdot \boldsymbol{v}(\cdot)-\mathscr{W}(\cdot)^{\top} \cdot \Theta^{-1} \cdot \boldsymbol{s}(\cdot)+\boldsymbol{Q}(\cdot)\right] \\
= & \varrho(\cdot) \boldsymbol{k}(\cdot) \cdot \boldsymbol{v}(\cdot)+\varrho(\cdot) \boldsymbol{g}(\cdot) \cdot \Theta^{-1} \cdot \boldsymbol{s}(\cdot)+\varrho(\cdot) r(\cdot) .
\end{aligned}
$$

Here $\varepsilon(\cdot)$ is the internal energy density, the mesoscopic absorption supply is $r(\cdot), \Theta$ the moment of inertia tensor, $\boldsymbol{q}(\cdot)$ the mesoscopic heat flux density, and $\boldsymbol{Q}(\cdot)$ is the heat flux density on $\mathscr{M}$.

According to the definition of the mesoscopic mass density (4) we obtain from the mesoscopic mass balance (13) a balance of the $\operatorname{MDF} f(\cdot)$ by inserting its 
definition:

$$
\begin{aligned}
\frac{\partial}{\partial t} f(\cdot)+\nabla_{x} \cdot[\boldsymbol{v}(\cdot) f(\cdot)] & +\nabla_{m} \cdot[\boldsymbol{u}(\cdot) f(\cdot)] \\
& +f(\cdot)\left[\frac{\partial}{\partial t}+\boldsymbol{v}(\cdot) \cdot \nabla_{x}\right] \ln \varrho(\boldsymbol{x}, t)=0 .
\end{aligned}
$$

Because this balance equation of the mesoscopic distribution function includes the macroscopic field of the mass density (5), it is not independent of the macroscopic mass balance defined on $\mathbb{R}^{3}$. Therefore the mesoscopic rate equation for the MDF contains already macroscopic quantities influencing its time rate. This can be interpreted as an influence of a "mean field" on the mesoscopic motion. As can be seen from (5) macroscopic quantities are defined by integration over the mesoscopic part. This should be investigated in more detail in the next section.

\section{Balances of Micropolar Media}

Introducing the macroscopic fields by integrating over the mesoscopic part of the mesoscopic space we can transform the local mesoscopic balances into local balances for micropolar media [19]. So the definition of the macroscopic material velocity $\boldsymbol{v}(\boldsymbol{x}, t)$ is

$$
\varrho(\boldsymbol{x}, t) \boldsymbol{v}(\boldsymbol{x}, t):=\int \varrho(\cdot) \boldsymbol{v}(\cdot) d \mathscr{M}
$$

and (13) results in the macroscopic mass balance, if we take

$$
\int \nabla_{m} \cdot[\boldsymbol{u}(\cdot) \varrho(\cdot)] d \mathscr{M}=0
$$

into consideration. This holds because of Gauss' or Stokes' theorem, and because $f(\cdot)$, restricted to the mesoscopic part, has a compact support [19].

The shape of the macroscopic balances by integrating (10) over the mesoscopic part is

$$
\begin{aligned}
\frac{\partial}{\partial t}\{\varrho(\boldsymbol{x}, t)[\phi(\boldsymbol{x}, t) \boldsymbol{v}(\boldsymbol{x}, t)+\boldsymbol{\Phi}(\boldsymbol{x}, t)]\} \\
\quad+\nabla_{x} \cdot\{\varrho(\boldsymbol{x}, t) \boldsymbol{v}(\boldsymbol{x}, t)[\phi(\boldsymbol{x}, t) \boldsymbol{v}(\boldsymbol{x}, t)+\boldsymbol{\Phi}(\boldsymbol{x}, t)]\} \\
=\boldsymbol{\Sigma}(\boldsymbol{x}, t)+\nabla_{x} \cdot \boldsymbol{S}(\boldsymbol{x}, t) .
\end{aligned}
$$


Here the following mesoscopic definitions of macroscopic quantities are introduced:

$$
\begin{aligned}
& \varrho(\boldsymbol{x}, t) \phi(\boldsymbol{x}, t):=\int \varrho(\cdot) \phi(\cdot) d \mathscr{M} \\
& \boldsymbol{\Sigma}(\boldsymbol{x}, t):=\int \boldsymbol{\Sigma}(\cdot) d \mathscr{M} \\
& \varrho(\boldsymbol{x}, t) \boldsymbol{\Phi}(\boldsymbol{x}, t):=\int \varrho(\cdot)[\phi(\cdot) \boldsymbol{v}(\cdot)+\boldsymbol{\Phi}(\cdot)] d \mathscr{M} \\
& +\varrho(\boldsymbol{x}, t) \phi(\boldsymbol{x}, t) \boldsymbol{v}(\boldsymbol{x}, t), \\
& \boldsymbol{S}(\boldsymbol{x}, t):=\int\{\boldsymbol{S}(\cdot)-\varrho(\cdot) \boldsymbol{v}(\cdot)[\phi(\cdot) \boldsymbol{v}(\cdot)+\boldsymbol{\Phi}(\cdot)]\} d \mathscr{M} \\
& -\varrho(\boldsymbol{x}, t) \boldsymbol{v}(\boldsymbol{x}, t)[\phi(\boldsymbol{x}, t) \boldsymbol{v}(\boldsymbol{x}, t)+\boldsymbol{\Phi}(\boldsymbol{x}, t)] .
\end{aligned}
$$

If $\phi(\cdot), \boldsymbol{\Phi}(\cdot), \boldsymbol{\Sigma}(\cdot)$, and $\boldsymbol{S}(\cdot)$ are specified according to (11) and (12) we obtain the macroscopic balance of mass. Those of momentum, angular momentum and energy follow in the same way by the specifications (14), (15), (17) to (20), and (23) to $(26)$.

Besides these balances an other one is essential: the entropy balance. Because of the second law which can be formulated only for the whole system the entropy balance is only interesting in its macroscopic form. It writes

$$
\begin{aligned}
& \frac{\partial}{\partial t}[\varrho(\boldsymbol{x}, t) \eta(\boldsymbol{x}, t)]+\nabla_{x} \cdot[\varrho(\boldsymbol{x}, t) \eta(\boldsymbol{x}, t) \boldsymbol{v}(\boldsymbol{x}, t)+\boldsymbol{\phi}(\boldsymbol{x}, t)] \\
& \quad=\zeta(\boldsymbol{x}, t)+\sigma(\boldsymbol{x}, t)
\end{aligned}
$$

$(\eta(\boldsymbol{x}, t)=$ specific entropy, $\boldsymbol{\phi}(\boldsymbol{x}, t)=$ entropy flux density, $\zeta(\boldsymbol{x}, t)=$ entropy supply, $\sigma(\boldsymbol{x}, t)=$ entropy production density). The second law is now expressed by the dissipation inequality

$$
\sigma(\boldsymbol{x}, t) \geq 0
$$

which has to be taken into account for writing down constitutive equations.

\section{Order Parameters and Constitutive Equations}

Using the set $\boldsymbol{m}$ of mesoscopic variables we can introduce the family of the macroscopic fields of order parameters which is defined by different moments of the 
MDF

$$
\begin{aligned}
& 1=\int f(\cdot) d \mathscr{M} \\
& \boldsymbol{A}(\boldsymbol{x}, t):=\int f(\cdot) \boldsymbol{m} d \mathscr{M} \\
& \boldsymbol{a}(\boldsymbol{x}, t):=\int f(\cdot) \overline{\boldsymbol{m m}} d \mathscr{M}, \\
& \underline{\boldsymbol{a}}_{4}(\boldsymbol{x}, t): = \int f ( \cdot ) \longdiv { \boldsymbol { m m m \boldsymbol { m } } } d \mathscr { M }, \\
& \underline{\boldsymbol{a}}_{N}(\boldsymbol{x}, t): = \int f ( \cdot ) \longdiv { \boldsymbol { m } \ldots N } \text { times } \ldots \boldsymbol{m} d \mathscr{M}, \quad \text { etc. }
\end{aligned}
$$

Here the symbol $\neg$ denotes the traceless symmetric part of the tensor in its argument [25].

These fields of order parameters describe macroscopically the mesoscopic state of the system introduced by $\boldsymbol{m}$ and its $\operatorname{MDF} f(\cdot)$. Consequently these fields are the link between the mesoscopic background description of the system and its extended description by additional macroscopic fields. An example for order parameter fields is given in Section 6. Here in liquid crystal theory the fields of order parameters are alignment tensors of different orders. Other examples for order parameter fields are micro-stress fields in solids or order fields in polycrystalline materials.

In a purely macroscopic phenomenological theory the order parameters are internal variables and relaxation equations for them are postulated [26, 27]. The mesoscopic background in mind equations of motion for these variables can be derived starting out with the differential equation (29) of the MDF. We multiply (29) with $\overline{m_{\mu_{1}} \cdots m_{\mu_{l}}}$, integrate over the manifold $\mathscr{M}$ and obtain

$$
\begin{aligned}
& \int \frac{\partial}{\partial t}\left[f(\cdot) \widetilde{m_{\mu_{1}} \cdots m_{\mu_{l}}}\right] d \mathscr{M} \\
& +\int \nabla_{x} \cdot\left[\boldsymbol{v}(\cdot) f(\cdot) \stackrel{m_{\mu_{1}} \cdots m_{\mu_{l}}}{ }\right] d \mathscr{M} \\
& +\int \overparen{m_{\mu_{1}} \cdots m_{\mu_{l}}} \nabla_{m} \cdot[\boldsymbol{u}(\cdot) f(\cdot)] d \mathscr{M} \\
& + \int f ( \cdot ) \longdiv { m _ { \mu _ { 1 } } \cdots m _ { \mu _ { l } } } d \mathscr { M } \\
& {\left[\frac{\partial}{\partial t} \ln \rho(\boldsymbol{x}, t)+\boldsymbol{v}(\cdot) \cdot \nabla_{x} \ln \rho(\boldsymbol{x}, t)\right]=0 .}
\end{aligned}
$$


We supose that the mesoscopic manifold $\mathscr{M}$ is time independent. Then the time derivative and the derivative with respect to position can be interchanged with the integration over $\mathscr{M}$. We split the mesoscopic velocity into the macroscopic velocity and the deviation from this average

$$
\boldsymbol{v}(\cdot)=\boldsymbol{v}(\boldsymbol{x}, t)+\delta \boldsymbol{v}(\cdot)
$$

The resulting equation of motion for the MDF reads

$$
\begin{aligned}
& \frac{\partial}{\partial t} \int f(\cdot) m_{\mu_{1}} \cdots m_{\mu_{l}} d \mathscr{M}
\end{aligned}
$$

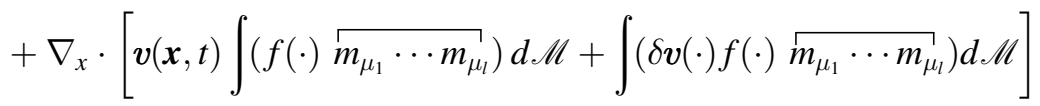

$$
\begin{aligned}
& + \int \longdiv { m _ { \mu _ { 1 } } \cdots m _ { \mu _ { l } } } \nabla _ { m } \cdot [ \boldsymbol { u } ( \cdot ) f ( \cdot ) ] d \mathscr { M } + \int f ( \cdot ) \longdiv { m _ { \mu _ { 1 } } \cdots m _ { \mu _ { l } } } d \mathscr { M } \\
& {\left[\frac{\partial}{\partial t} \ln \rho(\boldsymbol{x}, t)+\boldsymbol{v}(\cdot) \cdot \nabla_{x} \ln \rho(\boldsymbol{x}, t)\right]=0 .}
\end{aligned}
$$

Introducing the definition of the order parameters (43) we obtain

$$
\begin{aligned}
& \frac{\partial}{\partial t} \underline{\boldsymbol{a}_{l}}+\nabla_{x} \cdot\left[\boldsymbol{v}(\boldsymbol{x}, t) \underline{\boldsymbol{a}_{l}}+\int\left(\delta \boldsymbol{v}(\cdot) f(\cdot) \overline{m_{\mu_{1}} \cdots m_{\mu_{l}}}\right) d \mathscr{M}\right] \\
& +\int \overline{m_{\mu_{1}} \cdots m_{\mu_{l}}} \nabla_{m} \cdot[\boldsymbol{u}(\cdot) f(\cdot)] d \mathscr{M} \\
& +\underline{\boldsymbol{a}_{l}}\left[\frac{\partial}{\partial t} \ln \rho(\boldsymbol{x}, t)+\boldsymbol{v}(\cdot) \cdot \nabla_{x} \ln \rho(\boldsymbol{x}, t)\right]=0 .
\end{aligned}
$$

In case of a general mesoscopic manifold $\mathscr{M}$ this equation cannot be simplified further, but we will derive a simplified equation for the example of biaxial liquid crystals later.

For writing down constitutive equations a state space has to be introduced [28]. In mesoscopic theories there are different possibilities to do that: A state space of purely mesoscopic variables, a mixed one consisting of mesoscopic and macroscopic variables, or one containing only macroscopic variables can be introduced. In case of a state space of mesoscopic variables the well known macroscopic constitutive equations can be analogously reformulated on the mesoscopic state space [19], if the mesoscopic manifold $\mathscr{M}$ can be embedded in $\mathbb{R}^{3}$ (which is, of course, not generally the case). Such an embedding is possible for example in the mesoscopic theory for uniaxial liquid crystals. The mesoscopic background of the constitutive theory has been applied to the stress tensor of liquid crystals of uniaxial molecules [29]. The result is the order parameter dependence of viscosity coefficients, which cannot be obtained from a purely macroscopic theory. Another possibility is to introduce the 
mesoscopic definitions of the macroscopic quantities into the macroscopic balances. Consequently we obtain mesoscopically defined macroscopic quantities for which we can derive the class of the macroscopic constitutive equations by use of Liu's procedure $[30,31,32]$. In this case the simplest example of mesoscopically defined variables are the alignment tensors which are introduced additionally to the classical fields into the state space in order to take into account the dependence of material properties on the internal structure of the material. Mixed state spaces containing mesoscopic as well as macroscopic variables appear, if the dynamics of the order parameter fields is derived [33, 34].

\section{Example: Liquid Crystals of Biaxial Molecules}

As reported in Section 2 a prominent example for $\boldsymbol{m}$ is the microscopic director in liquid crystal theory for uniaxial molecules, because the orientation of such a molecule can be described by one direction represented by this microscopic director. In case of biaxial molecules we need two directions for describing the orientation of the molecule. A constraint between these two directors is the fixed angle between them, usually chosen to be $\pi / 2$. Therefore the orientation of a biaxial molecule is fixed by three parameters, two for the orientation of the first director, a third one for that of the second director [35]. Because of this constraint a two-director theory [36, 37] is clumsy to handle. Therefore it is advantageous to look for another set of mesoscopic variables for describing the orientation of biaxial liquid crystals. This is the item of the following section.

\subsection{Set of mesoscopic variables}

The orientation of a biaxial molecule is described by a proper orthogonal transformation $\mathbf{Q}$ which is defined on a triad of reference and which results in the actual orientation of the molecule [38]. Because $\mathbf{Q}$ is characterized by three parameters we may choose them as a set of mesoscopic variables. But this set is difficult to handle, and therefore we will replace it by another on. First of all we remember the

$\square$ Proposition (Euler-D'Alembert): Each proper orthogonal transformation on $\mathbb{R}^{3}$ can be described uniquely by a 3-dimensional unit vector $\boldsymbol{n}^{0} \in \mathbb{R}^{3},\left|\boldsymbol{n}^{0}\right|=1$, and an angle $\varphi \in[0, \pi]$, if we identify $\left(\boldsymbol{n}^{0}, \pi\right) \equiv\left(-\boldsymbol{n}^{0}, \pi\right)$ :

$$
\begin{aligned}
& \mathbf{Q} \cdot \mathbf{Q}^{\top}=\mathbf{Q}^{\top} \cdot \mathbf{Q}=\mathbf{1}, \quad \mathbf{Q} \cdot \boldsymbol{n}^{0}=\boldsymbol{n}^{0} \\
& 0=\boldsymbol{n}^{0} \cdot z^{0}=\boldsymbol{n}^{0} \cdot \mathbf{Q}^{\top} \cdot \mathbf{Q} \cdot z^{0}=: \boldsymbol{n}^{0} \cdot z^{0 *} \\
& z^{0} \cdot z^{0 *}=\cos \varphi
\end{aligned}
$$

According to this proposition all rotations around an arbitrary, but fixed axis of the direction $\left\{\boldsymbol{n}^{0},-\boldsymbol{n}^{0}\right\}$ are represented by the sets $\left(\boldsymbol{n}^{0}, \varphi\right)$ and $\left(-\boldsymbol{n}^{0}, \varphi\right)$. 
The three free parameters of an orthogonal transformation which are included in $\boldsymbol{n}^{0}$ and $\varphi$ can be represented by quaternions [39]. A quaternion $x \in \mathbb{M}^{2)}$ is defined as follows

$$
\begin{aligned}
& x=x_{0}+x_{1} i+x_{2} j+x_{3} k=: x_{0}+\tilde{x} \in \mathbb{M}, \\
& i^{2}=j^{2}=k^{2}=-1, \quad i j=k(\text { cycl. }), \quad j i=-k(\text { cycl. }) .
\end{aligned}
$$

The multiplication is as usual, but non-commutative. The part $x_{0} \in \operatorname{Re} \llbracket$ is called the real, and $\tilde{x} \in \operatorname{Im} \mathbb{H}$ is the imaginary part of the quaternion $x$.

We now introduce two (basis-dependent) mappings

$$
\begin{aligned}
& \boldsymbol{V}: \operatorname{Im} \boxplus \rightarrow \mathbb{R}^{3}, \tilde{a} \mapsto \boldsymbol{a}=\boldsymbol{V}(\tilde{a}), \\
& \underline{W}: \mathbb{H} \rightarrow \boldsymbol{R}^{4}, a \mapsto \underline{a}=\underline{W}(a) .
\end{aligned}
$$

The multiplication of two quaternions can be represented by use of these mappings as

$$
\begin{aligned}
& x y=(x y)_{0}+\tilde{x y}, \\
& (x y)_{0}=x_{0} y_{0}-\boldsymbol{V}(\tilde{x}) \cdot \boldsymbol{V}(\tilde{y}), \\
& \tilde{x y}=x_{0} \tilde{y}+\tilde{x} y_{0}+\boldsymbol{V}^{-1}(\boldsymbol{V}(\tilde{x}) \times \boldsymbol{V}(\tilde{y})) .
\end{aligned}
$$

Introducing the conjugate complex quaternion by

$$
x^{*}:=x_{0}-\tilde{x}
$$

we obtain for the square of its norm

$$
x x^{*}=x^{*} x=:|x|^{2}=x_{0}^{2}+\boldsymbol{V}^{2}(\tilde{x})=\underline{W}^{2}(x) .
$$

With regard to the Euler-D'Alembert proposition we now consider the special unit quaternion

$$
n=\cos (\varphi / 2)+\sin (\varphi / 2) \boldsymbol{V}^{-1}\left(\boldsymbol{n}^{0}\right) \in \mathbb{H}, \quad n^{*} n=1
$$

Because according to the Euler-D'Alembert proposition $0 \leq \varphi \leq \pi$ is valid, the real part of $n$ is always not negative, whereas the imaginary part depends on the direction of $\boldsymbol{n}^{0}$. Consequently all $n$ of negative real part do not represent a rotation, that means, not all unit 4-vectors $\underline{n}^{0}=\underline{W}(n)$ decribe a rotation. This is a disadvantageous situation because the unit 4-vectors $\underline{n}^{0}=\underline{W}(n) \in \mathbb{S}^{3}$ spanning the 3-dimensional sphere would be a suitable and easy set of mesoscopic variables.

${ }^{2)}$ The letter $\mathbb{T}$ has been chosen to honour R. W. Hamilton who invented these numbers in the middle of the $19^{\text {th }}$ century. 


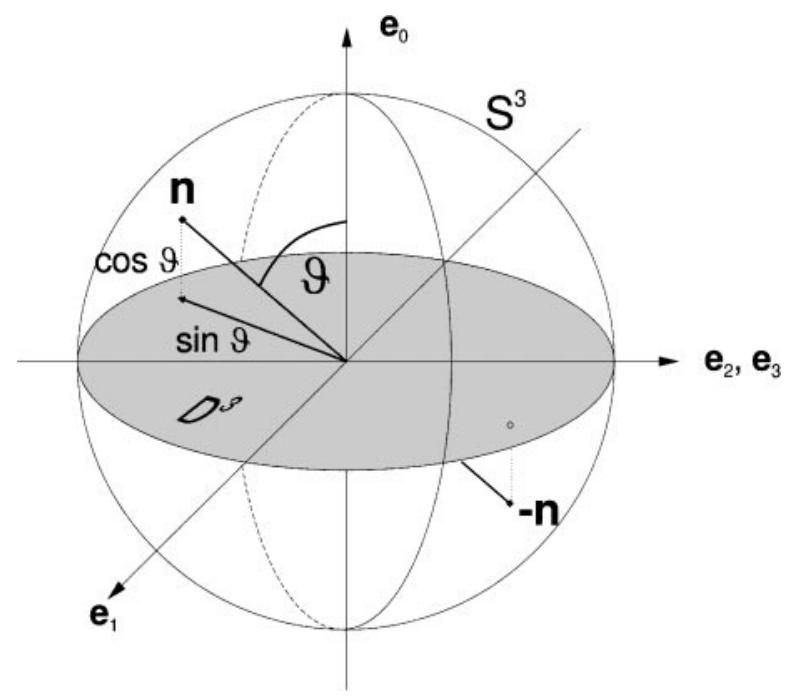

Fig. 1. The antipodes $\underline{n}^{0}$ and $-\underline{n}^{0}$ of the $\mathbb{S}^{3}$ represent by projection $\Pi$ the same rotation, characterized by points of the ball $\mathbb{D}^{3}$. Thus $\mathbb{S}^{3}$ is a universal covering of $\mathbb{D}^{3}$.

The solution of this dilemma is to consider $\mathbb{S}^{3}$ as a universal covering $\mathscr{C}$ of the space spanned by the vectors belonging to the imaginary parts of (60) which is the ball $\mathbb{D}^{3}$ of radius one (see Fig. 1)

$$
\begin{aligned}
& \mathscr{C}=<\mathbb{S}^{3}, \mathbb{D}^{3}, \Pi>, \Pi: \mathbb{S}^{3} \rightarrow \mathbb{D}^{3}, \\
& \underline{n}^{0}:=\cos \vartheta \boldsymbol{e}_{0}+\sin \vartheta \boldsymbol{n}^{0} \mapsto \Pi\left(\underline{n}^{0}\right):=[\operatorname{sgn} \cos \vartheta] \sin \vartheta \boldsymbol{n}^{0} .
\end{aligned}
$$

This procedure is now explained in more detail: According to the Euler-D-Alembert proposition the 3 -vector

$$
\boldsymbol{n}=\boldsymbol{V}(\tilde{n})=\sin (\varphi / 2) \boldsymbol{n}^{0} \in \mathbb{D}^{3}
$$

represents a rotation of angle $\varphi$ around the axis directed along $\boldsymbol{n}^{0}$. The imaginary part of the unit quaternion (60)

$$
\tilde{n}=\sin (\varphi / 2) \boldsymbol{V}^{-1}\left(\boldsymbol{n}^{0}\right) \in \operatorname{Im} \mathbb{H}, \quad\left|\boldsymbol{n}^{0}\right|=1 .
$$

is connected to this rotation by (63). Comparison of (63) with (62) makes evident, that then also $\Pi\left(\underline{n}^{0}\right)$ represents a rotation. Because of

$$
\Pi\left(-\underline{n}^{0}\right)=-\{\operatorname{sgn}[-\cos (\varphi / 2)]\} \sin (\varphi / 2) \boldsymbol{n}^{0}=\Pi\left(\underline{n}^{0}\right)
$$

we obtain the result that, by introducing the universal covering $\mathscr{C}$ (61), both the unit 4-vectors $\underline{n}^{0}$ and $-\underline{n}^{0}$ are mapped onto the same rotation, although only one of them 
(that of $\cos (\varphi / 2) \geq 0$ ) is representing one. Thus two unit 4 -vectors, $\underline{n}^{0} \in \mathbb{S}^{3}$, belong to each rotation.

If we now introduce $\underline{n}^{0}$ as the set of mesoscopic variables, (1) results in

$$
\left(\underline{n}^{0}, x, t\right) \in \mathbb{S}^{3} \times \mathbb{R}^{3} \times \mathbb{R}^{1} .
$$

Because $\underline{n}^{0}$ and $-\underline{n}^{0}$ belong to the same rotation the MDF (2) has the symmetry

$$
f\left(\underline{n}^{0}, \boldsymbol{x}, t\right)=f\left(-\underline{n}^{0}, \boldsymbol{x}, t\right) \equiv f(\bullet) .
$$

Before we can write down the local mesoscopic balances (10) we have to introduce the mesoscopic change velocity $\boldsymbol{u}(\cdot)$ which appear in (9) and (10).

\subsection{Angular and mesoscopic change velocity}

Like the mesoscopic set $\underline{n}^{0}$ the mesoscopic change velocity $\boldsymbol{u}(\cdot)$ is a 4 -vector defined on the mesoscopic space which indicates the change of the unit 4-vector $\underline{n}^{0}$. Therefore $\underline{u}$ is an element of the tangent space of the $\mathbb{S}^{3}$, and we have according to (56) and (58)

$$
\underline{n}^{0} \cdot \underline{u}=0=\left(n^{*} u\right)_{0}
$$

This relation can easily be verified by introducing

$$
u \equiv \dot{n} \rightarrow \underline{u}=\underline{W}(\dot{n}),
$$

and inserting (60) into (68). According to (60) and (69) we obtain

$$
\boldsymbol{V}(\tilde{u})=(1 / 2) \cos (\varphi / 2) \dot{\varphi} \boldsymbol{n}^{0}+\sin (\varphi / 2) \dot{\boldsymbol{n}}^{0}
$$

which consists of two parts belonging to the changes of the rotation angle and the rotation axis. This 3-vector will appear in the balance of energy. The gradient with respect to the set of mesoscopic variables reads now

$$
\nabla_{m} \cdot \boldsymbol{u} \equiv \nabla_{n} \cdot \underline{u} .
$$

The angular velocity $\omega$ is defined by the time dependent proper orthogonal transformations $\boldsymbol{Q}(t)$ in (48)

$$
\omega:=(1 / 2) \underline{\varepsilon}_{3}: \Omega, \quad \Omega:=Q^{\top} \cdot \dot{Q} .
$$

Here $\underline{\varepsilon}_{3}$ is the Levi-Civita tensor. Thus by introducing the tensor $\Theta$ of inertia the angular momentum and the rotation energy are

$$
\boldsymbol{n}:=\boldsymbol{\Theta} \cdot \boldsymbol{\omega}, \quad \varepsilon_{r}:=(1 / 2) \boldsymbol{\omega} \cdot \boldsymbol{\Theta} \cdot \boldsymbol{\omega}
$$

and we consider the special balances for liquid crystals of biaxial molecules in the next section. 


\subsection{Balances}

As we can see from (67) in comparison with (2) the argument $(\bullet)$ corresponds to the argument $(\cdot)$. Consequently the balance equations written down in section 3.2 are already those for biaxial liquid crystals, if we replace all arguments (.) by $(\bullet) \in \mathbb{S}^{3} \times \mathbb{R}^{3} \times \mathbb{R}^{1}$. The balances are now defined on the nematic space as a special case of the mesoscopic one, and some of the quantities have another interpretation than in Section 3.2.

The mixed tensor $\mathscr{T}$ is the stress tensor on $\mathbb{S}^{3}$ represented by the linear mapping

$$
\mathscr{T}: \mathbb{S}^{3} \rightarrow \mathbb{R}^{3}, \quad \underline{a} \mapsto \boldsymbol{a}=\mathscr{T}(\bullet) \cdot \underline{a}
$$

$\boldsymbol{g}(\bullet)$ is the 3-vector of angular momentum exerted by the external forces on the orientation, the 3-tensor $\boldsymbol{W}(\bullet)$ and the mixed tensor

$$
\mathscr{W}: \mathbb{S}^{3} \rightarrow \mathbb{R}^{3}, \quad \underline{a} \mapsto \boldsymbol{a}=\mathscr{W}(\bullet) \cdot \underline{a},
$$

are the analogues to $\boldsymbol{T}$ and $\mathscr{T}$ and are called spin tensors, $r(\bullet)$ is the absorption supply, $\boldsymbol{q}(\bullet)$ the heat flux density, and $\boldsymbol{Q}(\bullet)$ the heat flux density on the $\mathbb{S}^{3}$.

\subsection{Alignment tensors}

According to (43) the alignment tensors are introduced as the symmetric irreducible moments of the ODF

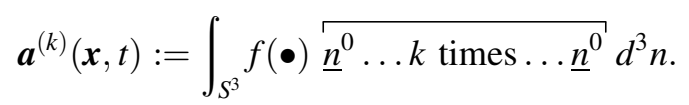

They are macroscopic fields describing the orientational order, being non-zero in the ordered phase and vanishing in the isotropic one. Therefore they are order parameters in the sense of the theory of phase transitions. Within a purely macroscopic phenomenological theory they are internal variables independent in non-equilibrium and depending in equilibrium on the equilibrium variables density and temperature.

The equations of motion for these internal variables can be derived in the way we derived the equations of motion for the order parameters (47) in the general mesoscopic theory

$$
\begin{aligned}
& \frac{\partial}{\partial t} \boldsymbol{a}_{l}+\nabla_{x} \cdot\left[\boldsymbol{v}(\boldsymbol{x}, t) \boldsymbol{a}_{l}+\int_{S^{3}}\left(\delta \boldsymbol{v}(\bullet) f(\bullet) \overline{n_{\mu_{1}}^{0} \cdots n_{\mu_{l}}^{0}}\right) d^{3} n\right] \\
& \quad+\int_{S^{3}} \overline{n_{\mu_{1}}^{0} \cdots n_{\mu_{l}}^{0}} \nabla_{n} \cdot[\boldsymbol{u}(\bullet) f(\bullet)] d^{3} n \\
& \quad+\boldsymbol{a}_{l}\left[\frac{\partial}{\partial t} \ln \rho(\boldsymbol{x}, t)+\boldsymbol{v}(\bullet) \cdot \nabla_{x} \ln \rho(\boldsymbol{x}, t)\right]=0 .
\end{aligned}
$$


For the manifold $\mathscr{M}$ being a unit sphere in $\mathbb{R}^{k}$ (here $S^{3}$ ) this equation can be simplified using [38, 25]

$$
\begin{aligned}
& \overline{n_{\mu_{1}}^{0} \cdots n_{\mu_{l}}^{0}} \nabla_{n} \cdot(\boldsymbol{u}(\bullet) f(\bullet)) \\
& \quad=\nabla_{n} \cdot\left(\boldsymbol{u}(\bullet) f(\bullet) \overline{n_{\mu_{1}}^{0} \cdots n_{\mu_{l}}^{0}}\right)-f(\bullet) \boldsymbol{u}(\bullet) \cdot \nabla_{n} \overline{n_{\mu_{1}}^{0} \cdots n_{\mu_{l}}^{0}} \\
& \quad=\nabla_{n} \cdot\left(\boldsymbol{u}(\bullet) f(\bullet) \overline{n_{\mu_{1}}^{0} \cdots n_{\mu_{l}}^{0}}\right)-l f(\bullet) \overline{u_{\mu_{1}}(\cdot) n_{\mu_{2}}^{0} \cdots n_{\mu_{l}}^{0}},
\end{aligned}
$$

where $\nabla_{n}$ denotes here the covariant derivative on the unit sphere.

The divergence on the unit sphere is transformed by use of Gauss' theorem, and the corresponding term vanishes after integration over the closed unit sphere. We introduce the abbreviation $\langle\cdots\rangle$ for the avarage with the orientational distribution function over the unit sphere and obtain

$$
\begin{aligned}
& \frac{\partial}{\partial t} a_{\mu_{1} \cdots \mu_{l}}(\boldsymbol{x}, t)+\nabla_{x} \cdot\left(\boldsymbol{v}(\boldsymbol{x}, t) a_{\mu_{1} \cdots \mu_{l}}(\boldsymbol{x}, t)\right) \\
& \quad+\nabla_{x} \cdot\left\langle\delta \boldsymbol{v}(\bullet) \overline{n_{\mu_{1}}^{0} \cdots n_{\mu_{l}}^{0}}\right\rangle-l\left\langle\overline{u_{\mu_{1}}(\bullet) n_{\mu_{2}}^{0} \cdots n_{\mu_{l}}^{0}}\right\rangle \\
& \quad+a_{\mu_{1} \cdots \mu_{l}}(\boldsymbol{x}, t)\left(\frac{\partial}{\partial t} \ln \rho(\boldsymbol{x}, t)+\boldsymbol{v}(\boldsymbol{x}, t) \cdot \nabla_{x} \ln \rho(\boldsymbol{x}, t)\right) \\
& \quad+\left\langle\delta \boldsymbol{v}(\bullet) \overline{n_{\mu_{1}}^{0} \cdots n_{\mu_{l}}^{0}}\right\rangle \cdot \nabla_{x} \ln \rho(\boldsymbol{x}, t)=0
\end{aligned}
$$

or

$$
\begin{aligned}
& \frac{\partial}{\partial t} a_{\mu_{1} \cdots \mu_{l}}(\boldsymbol{x}, t)+\boldsymbol{v}(\boldsymbol{x}, t) \cdot \nabla_{x} a_{\mu_{1} \cdots \mu_{l}}(\boldsymbol{x}, t) \\
& +\nabla_{x} \cdot\left\langle\delta \boldsymbol{v}(\bullet) \overline{n_{\mu_{1}}^{0} \cdots n_{\mu_{l}}^{0}}\right\rangle-l\left\langle\overline{u_{\mu_{1}}(\bullet) n_{\mu_{2}}^{0} \cdots n_{\mu_{l}}^{0}}\right\rangle \\
& +a_{\mu_{1} \cdots \mu_{l}}(\boldsymbol{x}, t) \frac{1}{\rho(\boldsymbol{x}, t)} \underbrace{\left[\frac{\partial}{\partial t} \rho(\boldsymbol{x}, t)+\nabla_{x}(\boldsymbol{v}(\boldsymbol{x}, t) \rho(\boldsymbol{x}, t))\right]}_{=0} \\
& +\left\langle\delta \boldsymbol{v}(\bullet) \overline{n_{\mu_{1}}^{0} \cdots n_{\mu_{l}}^{0}}\right\rangle \cdot \nabla_{x} \ln \rho(\boldsymbol{x}, t)=0 .
\end{aligned}
$$

To decompose the term $\left\langle\overline{u_{\mu_{1}}(\bullet) n_{\mu_{2}}^{0} \cdots n_{\mu_{l}}^{0}}\right\rangle$ we introduce the mesoscopic angular velocity which is different from $(72)$

$$
\omega(\bullet):=n \times u(\bullet) \rightarrow u(\bullet)=\omega(\bullet) \times n
$$

and write

$$
\boldsymbol{u}(\bullet)=\underline{\Omega}_{2}(\bullet) \cdot \boldsymbol{n},
$$

and by use of the Levi-Civita tensor we have

$$
\underline{\Omega}_{2}(\bullet):=-\underline{\varepsilon}_{3} \cdot \omega(\bullet) .
$$


Using this we define the mean angular velocity tensor which is also different from (72) by

$$
\underline{\boldsymbol{\Omega}}_{2}(\boldsymbol{x}, t):=\left\langle\underline{\boldsymbol{\Omega}}_{2}(\bullet)\right\rangle
$$

and decompose $\underline{\Omega}_{2}(\bullet)$ according to

$$
\underline{\boldsymbol{\Omega}}_{2}(\bullet)=\underline{\boldsymbol{\Omega}}_{2}(\boldsymbol{x}, t)+\delta \underline{\boldsymbol{\Omega}}_{2}(\bullet) .
$$

Using the material time derivative we obtain the differential equation in time of the alignment tensor family

$$
\begin{aligned}
& \frac { d } { d t } a _ { \mu _ { 1 } \cdots \mu _ { l } } - l \longdiv { \Omega _ { \mu _ { 1 } \lambda } ( \boldsymbol { x } , t ) a _ { \lambda \mu _ { 2 } \cdots \mu _ { l } } } \\
& =l\left\langle\overline{\delta \boldsymbol{\Omega}_{\mu_{1} \lambda}(\bullet) n_{\lambda}^{0} n_{\mu_{2}}^{0} \cdots n_{\mu_{l}}^{0}}\right\rangle-\nabla_{x} \cdot\left\langle\delta \boldsymbol{v}(\bullet) \overline{n_{\mu_{1}}^{0} \cdots n_{\mu_{l}}^{0}}\right\rangle \\
& +\left\langle\delta \boldsymbol{v}(\bullet) \overline{n_{\mu_{1}}^{0} \cdots n_{\mu_{l}}^{0}}\right\rangle \cdot \nabla_{x} \ln \rho(\boldsymbol{x}, t) .
\end{aligned}
$$

Here are some remarks on the general structure of the equation of motion (86) of the alignment tensor family: The left hand side is Jaumann's (co-moving and corotational) time derivative of a symmetric, $l$-th rank tensor describing the changes of $\underline{\boldsymbol{a}}_{l}$ which are noted by an observer travelling with velocity $\boldsymbol{v}(\boldsymbol{x}, t)$ and rotating with $\boldsymbol{\omega}(\boldsymbol{x}, t)$. The right hand side of (86) describes the non-trivial changes of $\underline{\boldsymbol{a}}_{l}$, that are the changes by alignment production and supply. If the peculiar velocities $\delta \boldsymbol{v}(\bullet)$ and $\delta \underline{\Omega}_{2}(\bullet)$ vanish, the alignment tensor $\underline{\boldsymbol{a}}_{l}$ will only change in time by the motion of the observer and not by production and supply. The right hand side of (86) contains averages with respect to $f(\bullet)$, and therefore it is a rather complicated function of all alignment tensors of the family. Thus we obtain an infinite system of partial differential equations which does not decouple in general. The coupling is due to the moments of the peculiar velocities $\delta \boldsymbol{v}(\bullet)$ and $\delta \underline{\Omega}_{2}(\bullet)$.

\section{Summary}

For describing complex materials additional fields besides the mass density, the momentum density, the angular momentum density, and the energy density are needed. There are different procedures to introduce these additional fields. They can be introduced by their balance equations defined on space-time, or they can be introduced as additional variables spanning together with the space-time variables the mesoscopic space. This second procedure is called the mesoscopic concept. The usual balances are now defined on the mesoscopic space and can be written down easily by using an extended Reynolds transport theorem. In connection with the mesoscopic mass density a statistical quantity, the mesoscopic distribution function, and its moments can be introduced. These moments are ordinary fields on space-time taking into account the mesoscopic back-ground introduced by the mesoscopic concept. 
The nematic space of biaxial molecules can be interpreted as special case of a mesoscopic space. Here the mesoscopic variables $\mathscr{M}$ are unit 4 -vectors spanning the $\mathbb{S}^{3}$. Then the nematic balance equations for mass, momentum, angular momentum, and energy can be written down for liquid crystals of biaxial molecules which represents a general Cosserat continuum. The connection of these balances to those of micropolar media is discussed. The theory of constitutive equations is out of scope of this sketch and will be treated elsewhere.

In the case of liquid crystals the moments of the MDF are called alignment tensors. They are the macroscopic (internal) variables characterizing the orientational order in the liquid crystaline phase. A hierarchy of coupled differential equations has been derived for them.

\section{Acknowledgement}

The authors are grateful for financial support provided by the VISHAY Company, D95085 Selb, Germany, and by the Sonderforschungsbereich 335 "Anisotrope Fluide" of the Deutsche Forschungsgemeinschaft. W.M. thanks G.A. Maugin for discussions during his stay at Laboratoire de Modélisation en Mécanique, Université Pierre et Marie Curie, Paris VI, which pushed forward the development of the concept of Mesoscopic Continuum Physics, and E. S. Şuhubi for a valuable hint.

\section{References}

[1] Muschik, W., Internal variables in non-equilibrium thermodynamics, J. Non-Equilib. Thermodyn. 15 (1990), 127-137.

[2] Maugin, G. A., Muschik, W., Thermodynamics with internal variables, J. Non-Equilib. Thermodyn. 19 (1994) 217-249, 250-289.

[3] De Gennes, P. G., Prost, J., The physics of liquid crystals, Clarendon Press, Oxford, 1993, Sect. 2.1.

[4] Maugin, G. A., The thermomechanics of plasticity and fracture, Cambridge University Press, Cambridge, 1992, Chap. 7.

[5] Truesdell, C., Noll, W., Non-linear field theories of mechanics, Encyclopedia of Physics, Vol. III/3, Springer Verlag, Berlin, 1965, Sect. 98.

[6] Ericksen, J. L., Anisotropic fluids. Arch. Rat. Mech. Anal. 4 (1960), 231.

[7] Leslie, F. J., Some constitutive equations for liquid crystals. Arch. Rat. Mech. Anal. 28 (1965), 265.

[8] Hess, S., Irreversible thermodynamics of nonequilibrium phenomena in molecular liquids and in liquid crystals, Z. Naturforsch. 30a (1975), 728.

[9] Maugin, G. A., Drouot R., Thermodynamic modelling of polymers in solution, Constitutive laws and microstructure Eds. Axelrad, D. R., Muschik, W. Springer Verlag, Berlin, (1980), p. 137-161.

[10] Cosserat, E., Cosserat, F., Sur la mécanique générale Acad. Sci. Paris 145 (1907), 1139.

[11] Cosserat, E., Cosserat, F., Théorie des corps déformable, Hermann, Paris, 1909.

[12] Eringen, A. C., Şuhubi, E. S., Nonlinear theory of simple microelastic solids I and II, Int. J. Engng. Sci. 2 (1964) 189, 389.

[13] Eringen, A. C., Kafadar, C. B. Polar field theories, Continuum physics Ed. Eringen, A. C. Vol. IV, p. 1-73, Academic Press, New York, 1976.

[14] Capriz, G., Continua with microstructure, Springer-Verlag, Berlin, 1989.

[15] Eringen, A. C., Lee, J. D., Liquid crystals and ordered fluids, Eds. Johnson, J. F., Porter, R. S. Vol. 2, p. 315-330, Plenum Press, New York, 1974. 
[16] Stephen, M. J., Straley, J. P., Physics of liquid crystals, Rev. Mod. Phys. 46 (1974), 617.

[17] Eringen, A. C., Liquid crystals and ordered fluids, Eds. Johnson, J. F., Porter, R. S., Vol. 3, p. 443-474, Plenum Press, New York, 1974.

[18] Condiff, D. W., Brenner, H., Transport mechanics in systems of orientable particles, Phys. Fluids 12 (1969), 539.

[19] Blenk, S., Ehrentraut, H., Muschik, W., Statistical foundation of macroscopic balances for liquid crystals in alignment tensor formulation, Physica A 174 (1991), 119-138.

[20] Blenk, S., Ehrentraut, H., Muschik, W., A continuum theory for liquid crystals describing different degrees of orientational order, Liquid Cryst. 14 (1993), 1221-1226.

[21] Müller, W. H., Muschik, W., Bilanzgleichungen offener mehrkomponentiger systeme I: Massen- und Impulsbilanzen, J. Non-Equilib. Thermodyn. 8 (1983), 29-46.

[22] Muschik, W., Müller, W. H., Bilanzgleichungen offener mehrkomponentiger systeme II: Energie- und Entropiebilanz, J. Non-Equilib. Thermodyn. 8 (1983), 47-66.

[23] Blenk, S., Muschik, W., Orientational balances for nematic liquids crystals, J. NonEquilib. Thermodyn. 16 (1991), 67-87.

[24] Blenk, S., Ehrentraut, H., Muschik, W., Orientation balances for liquid crystals and their representation by alignment tensors, Mol. Cryst. Liqu. Cryst. 204 (1991), 133-141.

[25] Ehrentraut, H., Muschik, W., On symmetric irreducible tensors in $d$ dimensions, ARI 51 (1998), 149-159.

[26] De Groot, S. R., Mazur, P., Non-equilibrium thermodynamics, North-Holland, Amsterdam, 1962, Ch.X 5.

[27] Verhás, J., Thermodynamics and Rheology, Akadémiai kiadó, Budapest, 1997, Ch. VI.

[28] Muschik, W., Aspects of non-equilibrium thermodynamics, World Scientific, Singapore, 1990, Sect. 6.3.

[29] Ehrentraut, H., Hess, S., Viscosity coefficients of partially aligned nematic and nematic discotic liquid crystals, Phys. Rev. E 51 (1995), 2203-2212.

[30] Liu, I-S., Method of Lagrange multipliers for exploitation of the entropy principle, Arch. Rat. Mech. Anal. 46 (1972), 131-148.

[31] Blenk, S., Ehrentraut, H., Muschik, W., Macroscopic constitutive equations for liquid crystals induced by their mesoscopic orientation distribution, Int. J. Engng. Sci. 30 (1992), 1127-1143.

[32] Muschik, W., Ehrentraut, H., An amendment to the second law, J. Non-Equilib. Thermodyn. 21 (1996), 175-192, Appendix B.

[33] Ehrentraut, H., Muschik, W., Papenfuss, C., Mesoscopically derived orientation dynamics of liquid crystals, J. Non-Equilib. Thermodyn. 22 (1997), 285-298.

[34] Muschik, W., Papenfuss, C., Ehrentraut, H. Alignment tensor dynamics induced by the mesoscopic balance of the orientation distribution function, Proc. Estonian Acad. Sci. Phys. Math. 46 (1997), 94-101.

[35] Ehrentraut, H., Muschik, W., Balance laws and constitutive equations of microscopic rigid bodies: a model for biaxial liquid crystals, Mol. Cryst. Liq. Cryst. 262 (1995), 561568.

[36] Jacobsen, E. A., Swift, J., Hydrodynamics of biaxial nematic liquid crystals, Mol. Cryst. Liq. Cryst. 78 (1981), 311-317.

[37] Carlsson, T., Leslie, F. M., Behaviour of biaxial nematics in the presence of electric and magnetic fields, Liquid Crystals 10 (1991), 325-340.

[38] Ehrentraut, H., A unified mesoscopic continuum theory of uniaxial and biaxial liquid crystals, Wissenschaft- und Technik Verlag, Berlin, 1996, ISBN 3-89685-406-2.

[39] Harauz, G., Representation of rotations by unit quaternions, Ultramicroscopy 33 (1990), 209-213.

Paper received: 2000-5-22

Paper accepted: 2000-6-30 
Professor Dr. Wolfgang Muschik ${ }^{3)}$

Institut für Theoretische Physik

PN7-1

Technische Universität Berlin

Hardenbergstr. 36

10623 Berlin

Germany

Dr. Christina Papenfuss

Institut für Mechanik

Technische Universität Berlin

Hardenbergstr. 36

10623 Berlin

Germany

Dr. Harald Ehrentraut

Institut für Mechanik

Technische Universität Darmstadt

64289 Darmstadt

Germany

${ }^{3)}$ Corresponding author: muschik@ @hysik.tu-berlin.de

J. Non-Equilib. Thermodyn. $\cdot 2000 \cdot$ Vol $25 \cdot$ No. 2

Bereitgestellt von | Technische Universität Berlin 